\title{
Effects of chemical treatment on barrier height and ideality factors of Au/GaN Schottky diodes
}

\author{
M. Diale*, F.D. Auret \\ Department of Physics, University of Pretoria, Lynwood Road, Pretoria 0002, South Africa
}

\section{A R T I C L E I N F O}

PACS:

73.30. $+\mathrm{y}$

$79.40 .+\mathrm{z}$

Keywords:

Surface treatment

Schottky contact

\begin{abstract}
A B S T R A C T
We have studied $\mathrm{Au} / \mathrm{n}$-GaN Schottky barrier diodes. GaN surfaces have been prepared by cleaning in $\mathrm{HCl}$ and $\left(\mathrm{NH}_{4}\right)_{2} \mathrm{~S}$ prior to metal deposition. The zero-biased barrier heights and ideality factors obtained from the current-voltage characteristics differ from diode to diode, although all the samples were prepared identically. The statistical analysis for the reverse bias $C-V$ data yielded mean value of $(1.35 \pm 0.04) \mathrm{eV}$ for Schottky barrier height of $\mathrm{HCl}$ treated sample and $(1.20 \pm 0.03) \mathrm{eV}$ for $\left(\mathrm{NH}_{4}\right)_{2} \mathrm{~S}$ sample, where 9 dots were considered from each cleaning method. It was found that the barrier height values obtained from the $C^{-2}-V(1.43 \mathrm{eV})$ and $I-V$ characteristics $(0.89 \mathrm{eV})$ are different from each other by $0.54 \mathrm{eV}$. The inhomogeneous barrier heights were found to be related to the effect of the high series resistance on diode parameters (Akkiliç et al., 2004) [1].
\end{abstract}

(c) 2009 Elsevier B.V. All rights reserved.

\section{Introduction}

Rectifying contacts with low leakage currents and high barrier height are required for the successful fabrication of GaN-based devices. Schottky barrier diodes (SBD) are the choice structure for many semiconductor devices, including microwave diodes, fieldeffect transistors and photodiodes [2-4]. Their technological importance requires a full understanding of the nature of the electrical characteristics of SBDs. It is well known that SBD has a thin layer of an oxide between the metal and the semiconductor, which cannot be removed by conventional chemical cleaning. Such an oxide converts the diode to metal-insulator-semiconductor (MIS) and usually influences the electrical characteristics of the diode, causing a change in the interfacial charge with bias, giving rise to an electric field at the interfacial layer between the metal and the semiconductor [5,6]. The oxide layer reduces the barrier height and consequently increases the series resistance.

Generally, the forward biased current-voltage $(I-V)$ characteristics are linear in the semi-logarithmic scale at low voltages, but deviate considerably from linearity due to the effects of series resistance, $R_{S}$ resulting from the presence of the thin oxide layer and other surface contaminants. The series resistance is only effective in the curvature downward region or non-linear region of the forward $I-V$ characteristics at sufficiently high voltages. The concavity of the current-voltage characteristics at higher voltages increases with increasing series resistance. Increasing series resistance decreases the barrier height and this result in

\footnotetext{
* Corresponding author. Tel.: +2712420 4418; fax: +27123625288.

E-mail address: mmantsae.diale@up.ac.za (M. Diale).
}

non-ideal current-voltage characteristics. Other parameters such as the ideality factor, $n(V)$ and zero bias barrier height, $\Phi_{b, 0}$ are effective in both the linear and the non-linear regions of the $I-V$ curve, accompanying the changes in the Schottky barrier height (SBH) [7]. The effect of the series resistance between the depletion region and the ohmic contact of the neutral region of the semiconductor bulk causes the $I-V$ characteristics of the metalsemiconductor contact to deviate from the expected [8].

The interface states at the metal-semiconductor junction play a vital role in evaluating the Schottky barrier height and the ideality factor. These manifest themselves as deviations from the ideal Schottky barrier formation and are localized within a few atomic layers of the intimate metal-semiconductor contact with energies which fall inside the forbidden gap. Bardeen showed that such charge accumulated at the metal-semiconductor contact reduces the effective potential difference between the semiconductor and the metal contact [9]. Interface states arise from semiconductor surface states due to discontinuity in the lattice potential, metal-induced-gap states due to wave-function tunneling from the metal into the semiconductor, surface states due to contamination and defects; and any new compounds formed as a result of the interaction of the metal and the semiconductor.

A study of the importance of series resistance in calculating the characteristic parameters of Si Schottky contacts was done by Aydin et al. [1], obtaining their estimations from determination of interface states density distribution from the analysis of the current-voltage measurements. Kampen and Monch studied the barrier heights of different metals on GaN using metal-induced gap states (MIGS) and the electronegativity model, concluding that the experimental values of the barrier height are excellently reproduced by the theoretical predictions, which follow from 
physical MIGS and the electronegativity concept [10]. A review of metal-contact technology has revealed the importance of surface preparation prior to metal deposition [11]. In this study, two different surface chemicals were used to treat GaN surface prior to metal deposition. The effects of chemical treatments on Schottky characteristics were investigated using capacitance-voltage $(C-V)$ and current-voltage $(I-V)$ characteristics. The average barrier height for the diodes was 1.43 and $1.20 \mathrm{eV}$ for $C-V$; and 0.81 and 0.89 for $I-V$ measurements, respectively.

\section{Experimental}

For this investigation, we have used GaN samples with carrier density of $1 \times 10^{17} \mathrm{~cm}^{-3}$, obtained from TDI. Before contact fabrication, samples were cleaned using trichloroethylene (TEC), Isopropanol and $\mathrm{HCl}: \mathrm{HNO}_{3}$ aquaregia. Each of these samples was finally etched in 1:1 HCl: $\mathrm{H}_{2} \mathrm{O}$ (sample 1 ) and $\left(\mathrm{NH}_{4}\right)_{2} \mathrm{~S}$ (sample 2), respectively. Using patterned surface, $\mathrm{Ti} / \mathrm{Al} / \mathrm{Ni} / \mathrm{Au}(150 / 2200 / 400 /$ $500 \AA$ ) ohmic contacts were deposited by electron-beam and annealed in ultra pure $\mathrm{Ar}$ for $5 \mathrm{~min}$ at $500^{\circ} \mathrm{C}$. Thereafter, $\mathrm{Au}$ Schottky contacts, $0.25 \mathrm{~mm}$ thick, were deposited in the resistive evaporator at room temperature. The values of zero-biased barrier height and ideality factor were determined from $I-V$ and $C-V$ measurements at room temperature and corrected afterwards for the effect of series resistance.

\section{Results and discussion}

In Schottky diodes, the depletion layer capacitance can be expressed as [2]

$C^{-2}=\frac{2\left(V_{b i}-V_{A}\right)}{q \varepsilon_{S} A^{2} N_{D}}$

where $A$ is the area of the diode, $V_{b i}$ the diffusion potential at zero bias and is determined from the extrapolation of the linear $C^{-2}-$ $V$ plot to the $V$ axis and $V_{A}$ is the applied voltage. The value of the barrier height can be obtained from the relation:

$\Phi_{b, 0}(C-V)=V_{b i}+V_{0}$ where $V_{0}$ is the potential difference between the bottom of the conduction band and the Fermi level; and can be calculated knowing the donor concentration $N_{D}$ obtained from the following relation:

$V_{0}=(k T) \ln \left(\frac{N_{C}}{N_{D}}\right)$

where $N_{C}=4.6 \times 10^{16} \mathrm{~cm}^{-3}$ is the effective density of states in the conduction band [3].

Nine dots with the same diameter $(0.25 \mathrm{~mm})$ on each sample were evaluated. Fig. 1 shows the reverse bias $C^{-2}-V$ characteristics for one diode from sample 1 and sample 2, respectively. For these particular diodes on samples 1 and 2, the $C-V$ barrier heights are 1.43 and $1.20 \mathrm{eV}$, respectively. The carrier concentration of $1.9 \times 10^{16}$ and $2.4 \times 10^{16} \mathrm{~cm}^{-3}$ from the reverse bias $C^{-2}-V$ plots was obtained for samples 1 and 2 . The $C-V$ barrier heights ranged from 1.28 to $1.50 \mathrm{eV}$ for sample 1 and from 1.14 to $1.25 \mathrm{eV}$ for sample 2. The statistical analysis for the $C-V$ data yielded SBH mean value of $1.35 \pm 0.04 \mathrm{eV}$ for sample 1 dots and SBH mean value of $1.20 \pm 0.03 \mathrm{eV}$ for sample 2 .

In Schottky barrier diodes, the barrier height depends on the voltage and surface conditions prior to metal deposition. The surface condition includes the thickness of the interfacial oxide, which affects the current-transport mechanisms. These include the thermionic emission, which is characterized by ideality close to unity and thermionic field emission and field emission. These mechanisms are affected by series resistance, tunneling and generation recombination in the depletion region. Table 1 gives the summary of the electrical characteristics of the diodes.

For a Schottky contact with series resistance, the net current of the device is due to thermionic emission and it is written as [2]

$I=I_{0} \exp \left(-\frac{q\left(V_{A}-I R_{S}\right)}{n k T}\right)$

where the saturation current, $I_{0}$ is expressed as

$I_{0}=A A^{*} T^{2} \exp \left(-\frac{q \Phi_{b, 0}}{k T}\right)$

where $q$ is the electron charge, $A^{*}$ is the effective Richardson constant and is equal to $26 \mathrm{~A} / \mathrm{cm}^{2} \mathrm{~K}^{2}$ for n-type $\mathrm{GaN}$ [12], $A$ is the

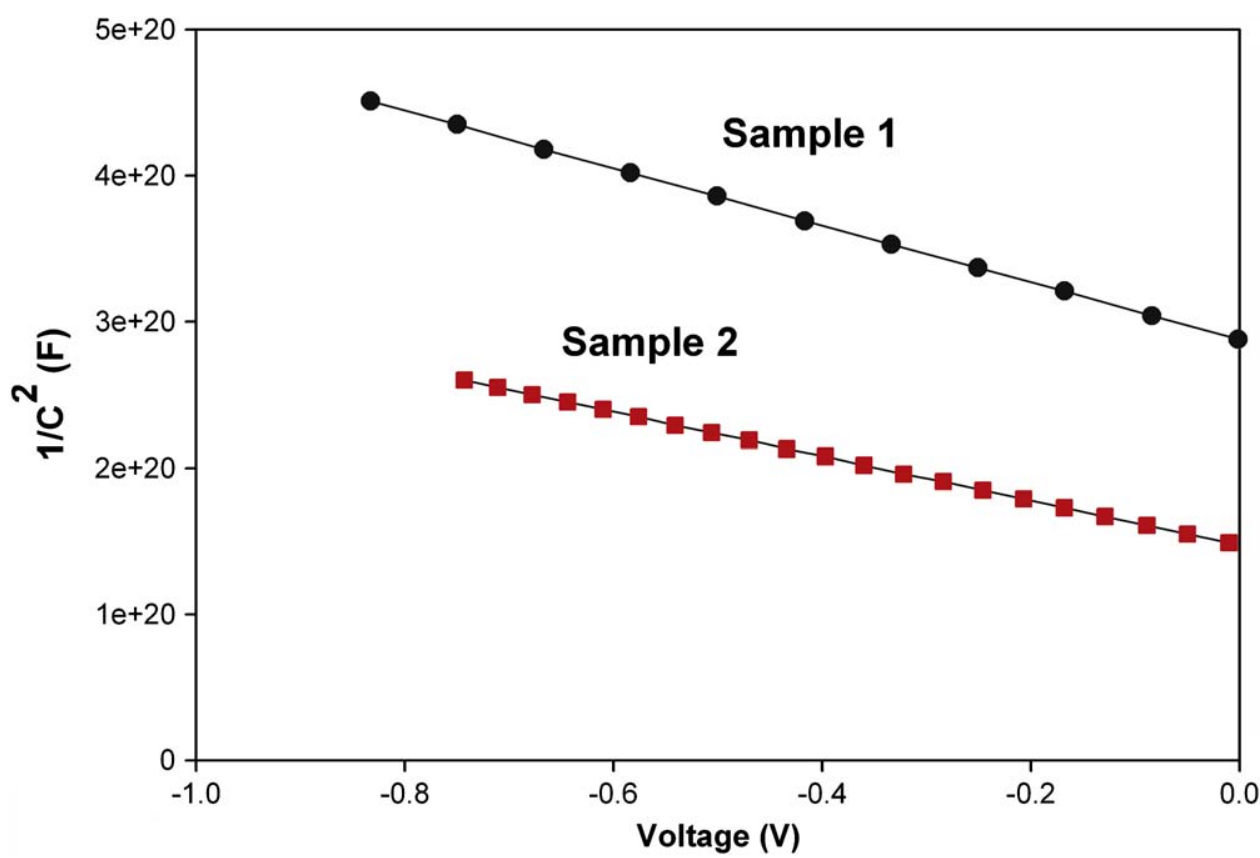

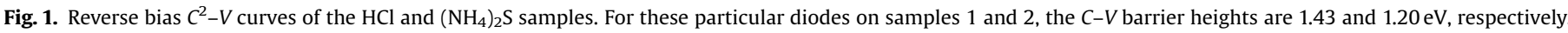


diode area, $T$ is the absolute temperature, $k$ Boltzmann constant, $n$ the ideality factor of the SBD and $\Phi_{b .0}$ the zero bias barrier height. When $V_{A} \geq 3 k T / q$, the extrapolated current, $I_{0}$ and the zero bias barrier height can be expressed as

$\Phi_{b, 0}=\frac{k T}{q} \ln \left(\frac{A^{*} A T^{2}}{I_{0}}\right)$

and the ideality factor from Eq. (4) can be written as

$n=\frac{q}{k T} \frac{d V}{d(\ln I)}$

The ideality factor of the SBD, $n$ is a measure of the conformity of the diode to pure thermionic emission. From Fig. 2, currenttransport mechanisms displayed are thermionic emission and the series resistance effect at high voltages. The values of the ideality factor, $n$ and the barrier height, $\Phi_{b}$ were calculated from the forward $I-V$ characteristics according to Eqs. (6) and (7). For sample 1 the barrier height, $\Phi_{b .0}$ ranged from 0.79 to $0.89 \mathrm{eV}$ and the ideality factor $n$ ranged from 1.02 to 1.17 . Sample $2 \Phi_{b .0}$ values ranged from 0.71 to $0.85 \mathrm{eV}$ and the $n$ from 1.31 to 1.36 . The statistical analysis yielded mean values of $0.84 \pm 0.05 \mathrm{eV}$ for the $1.06 \pm 0.50$ for barrier height and ideality factor of sample 1 (9 dots), respectively, and the mean values of $0.80 \pm 0.01 \mathrm{eV}$ and $1.34 \pm 0.20$ ( 9 dots) for sample 2 diodes. Ideality factors

Table 1

Values obtained experimentally from the current-voltage characteristics of the $\mathrm{Au} / \mathrm{GaN}$ Schottky diodes.

$\begin{array}{lc}\text { Sample } 1 & \\ n & 1.17 \\ R_{s}(\Omega) & 22.3 \\ \Phi_{b, c}(\mathrm{eV}), C-V & 1.43 \\ \Phi_{b, 0}(\mathrm{eV}), I-V & 0.82 \\ \text { Sample 2 } & \\ n & 1.89 \\ R_{s}(\Omega) & 17.1 \\ \Phi_{b, c}(\mathrm{eV}), C-V & 1.20 \\ \Phi_{b, 0}(\mathrm{eV}), I-V & 0.71\end{array}$

The difference in series resistance for the samples 1 and 2 is due to the surface state after different chemical treatment. above unity has been attributed to interface states due to thin oxide layer between the metal and the semiconductor, including other contaminants, tunneling currents in highly doped semiconductors, image-force lowering of the Schottky barrier in electric field at the interface and generation-recombination currents within the depletion region [2]. Our previous results have shown $\mathrm{S}$ and $\mathrm{Cl}$ residues onto $\mathrm{GaN}$ after cleaning in $\mathrm{HCl}$ and $\left(\mathrm{NH}_{4}\right)_{2} \mathrm{~S}$ using Auger electron spectroscopy (AES) and X-ray photoelectron spectroscopy (XPS) [13]. The work done on GaAs and GaP nitridation has shown anion exchange where a thin layer of Ga-N was formed on each of the materials [14]. Surface $\mathrm{Ga}-\mathrm{N}$ in turn passivates the GaAs and GaP, affecting the $I-V$ and $C-V$ characteristics of these materials. In addition, the work done by Liu et al. has shown that the Ga peak becomes larger when samples are cleaned in $\left(\mathrm{NH}_{4}\right)_{2} \mathrm{~S}$ than in $\mathrm{HF} / \mathrm{HCl}$ [15]. Furthermore, $\left(\mathrm{NH}_{4}\right)_{2} \mathrm{~S}$ has been found to reduce the barrier height on GaN, and preventing re-oxidation of the surface [16]. We suggest that there exist $\mathrm{Ga}-\mathrm{Cl}$ and $\mathrm{Ga}-\mathrm{S}$ on sample 1 and sample 2, respectively. Previous XPS results have shown that as-grown GaN surface has oxides in the form of $\mathrm{Ga}_{2} \mathrm{O}_{3}$ and $\mathrm{GaOH}$. In addition, while rinsing $\mathrm{GaN}$ in water, addition of $\mathrm{OH}$ to $\mathrm{GaN}$ to form the $\mathrm{GaOH}$, may occur, and be part of sticking surface water that may contribute to interface states [17].

The values of $R_{s}$ and $\Phi_{b .0}$ for both samples 1 and 2 were obtained as $0.82 \mathrm{eV}$ and $22.3 \Omega$; and $0.71 \mathrm{eV}$ and $17.0 \Omega$, respectively. As mentioned above, the barrier height values of 1.43 and $1.20 \mathrm{eV}$ for samples 1 and 2 were obtained from the $C^{-2}-V$ plots, respectively. These barrier height values obtained from the $C^{-2}-V$ $(1.43 \mathrm{eV})$ and $I-V$ characteristics $(0.89 \mathrm{eV})$ are different from each other by $0.54 \mathrm{eV}$. We attribute the difference between the $I-V$ and $C-V$ barrier height in the metal-semiconductor to SBH inhomogeneity. This is the fact that the barrier heights of the diodes on the same sample differs from diode to diode and at different positions on the same diode. The measured $I-V$ barrier height is significantly lower than the weighted arithmetic average of the SBHs. On the other hand, the $C-V$ measured barrier height is influenced by the distribution of charge at the depletion region follows the weighted arithmetic average of the barrier height inhomogeneity; hence the $\mathrm{BH}$ determined by $C-V$ is close to the weighted arithmetic average of the barrier heights. Therefore, the

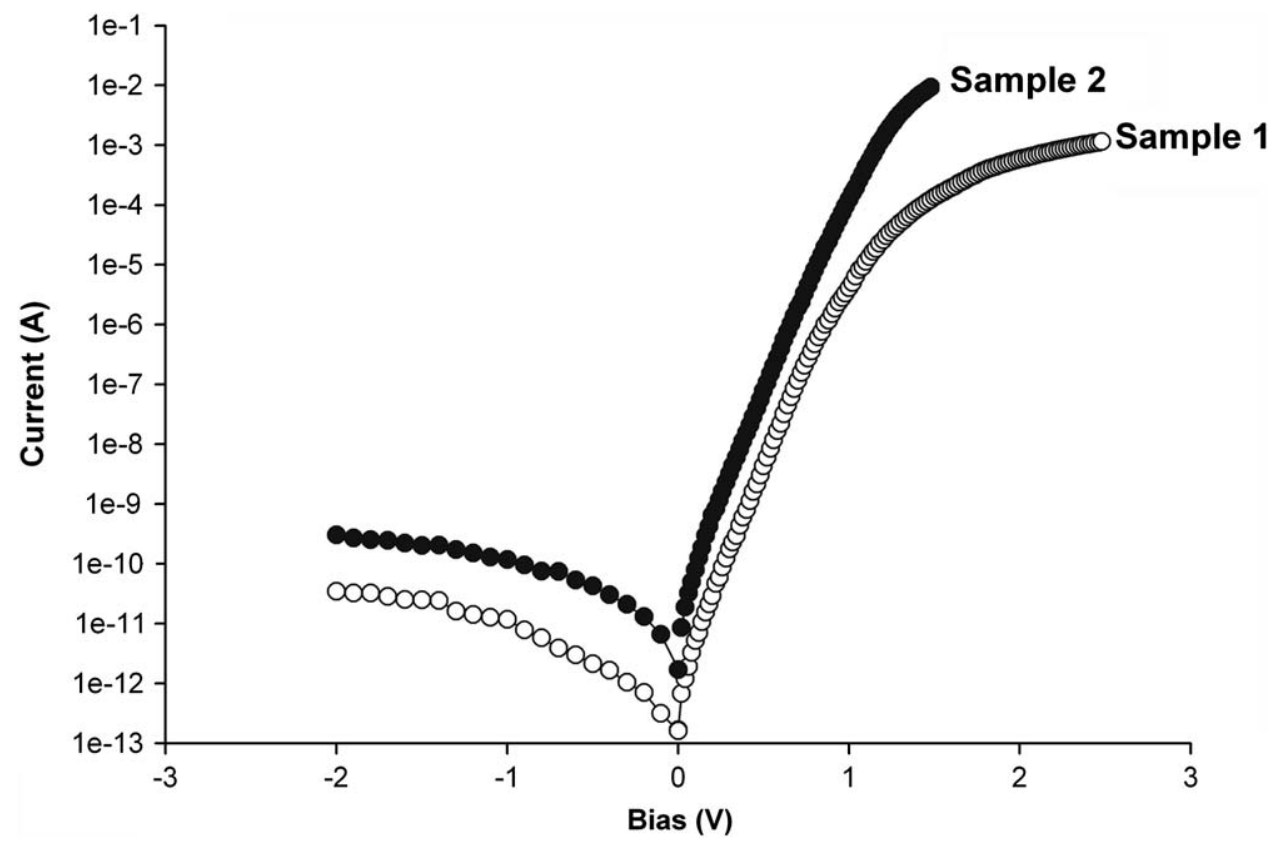

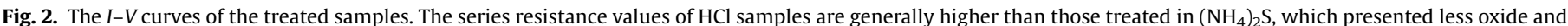
reduced barrier height. 
barrier height determined from zero bias intercept assuming thermionic emission as current transport mechanism is well below the measured $\mathrm{BH}$ and the weighted arithmetic average of the barrier heights $[18,19]$. Furthermore, the surface damage at the metal-semiconductor interface affects the $I-V$ measurements because defects may act as recombination centers for trapassisted tunneling currents. $C-V$ measurements are generally less prone to interface states, so that the determined barrier height is considered more reliable, though the depletion width can be altered by the interface defects if they are deeper into the space charge region [20].

\section{Summary}

In conclusion, we have fabricated Au/n-GaN SBDs using different cleaning procedures. From the current-voltage characteristics, we obtained the values of ideality factor, SBH and $R_{S}$ for the samples. The $I-V$ characteristics are near ideal with thermionic emission as the dominant current transport mechanism. Furthermore, $\mathrm{HCl}$ treated samples behave like a MIS diode due to the amount of oxide remaining on the surface after treatment. The series resistance values of $\mathrm{HCl}$ samples are generally higher than those treated in $\left(\mathrm{NH}_{4}\right)_{2} \mathrm{~S}$, which presented less oxide and reduced barrier height, in agreement with published results. Most published results on GaN have only reported their findings without specifics on current transport mechanism. Thus further work is needed for the investigation ideality factor far above unity, which will need the knowledge of the oxide layer thickness on $\mathrm{GaN}$, effects of passivation of GaN surface on electrical characteristics, and analysis of barrier height inhomogeneities on the rectifying diode characteristics on GaN.

\section{Acknowledgment}

The authors gratefully acknowledge financial assistance from the South African National Research Foundation.

\section{References}

[1] K. Akkilic, M.E. Aydin, A. Türüt, Physica Scripta 70 (2004) 364

[2] E.H. Rhoderick, R.H. Williams, Metal-Semiconductor Contacts, Clarendon Press, Oxford, 1988.

[3] S.M. Sze, Physics of Semiconductor Devices, second ed., Wiley, New York, 1981.

[4] L. Wang, M.I. Nathan, T.-H. Lim, M.A. Khan, Q. Chen, Applied Physics Letters 68 (9) (1996) 1267.

[5] P. Hanselaer, W.H. Laflere, R.L. Meirhaeghe, F. Cardon, Applied Physics Letters 56 (1984) 2309.

[6] E. Monroy, F. Calle, J.L. Pau, E. Muńoz, F. Omnes, Electronic Letters 36 (25) (2000) 2096.

[7] J.C. Card, E.H. Rhoderick, Journal of Applied Physics D 4 (1971) 1589.

[8] M.E. Aydin, K. Akkiliç, T. Kiliçoglu, Applied Surface Science 225 (2004) 1304.

[9] J. Bardeen, Physical Review 71 (1947) 771.

[10] T.U. Kampen, W. Monch, Applied Surface Science 117/118 (1997) 388.

[11] Q.Z. Liu, S.S. Lau, Solid State Electronics 42 (1998) 677.

[12] A.C. Schmidt, A.T. Ping, M. Asif Khan, Q. Chen, J.W. Yang, I. Adesida, Semiconductor Science and Technology 11 (1996) 1464

[13] M. Diale, F.D. Auret, N.G. van der Berg, R.Q. Odendaal, W.D. Roos, Applied Surface Science 246 (2005) 279.

[14] G. Bruno, Applied Surface Science 235 (2004) 239.

[15] J. Liu, B. Shen, Y.G. Zhou, H.M. Zhou, M.J. Wang, Z.W. Zheng, B. Zhang, Y. Shi, Y.D. Zheng, Optical Materials 23 (2003) 133.

[16] X.A. Cao, S.J. Pearton, G. Dang, A.P. Zhang, F. Ren, J.M. Van Hove, Applied Physics Letters 75 (1999) 4130.

[17] M. Diale, F.D. Auret, N.G. van der Berg, R.Q. Odendaal, W.D. Roos, Surface and Interface Analysis 37 (2005) 1158.

[18] J.H. Werner, H.H. Guttler, Journal of Applied Physics 69 (3) (1991) 1552.

[19] R.T. Tung, A.F.J. Levi, J.P. Sullivan, F. Schrey, Physical Review Letters 66 (1) (1971) 72.

[20] C. Fontaine, T. Okumura, K.N. Tu, Journal of Applied Physics 54 (1983) 1404. 